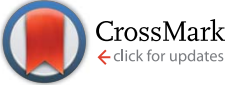

Cite this: RSC Adv., 2016, 6, 66516

\title{
Incorporation of graphene into silica-based aerogels and application for water remediation $\uparrow$
}

\author{
D. Loche, ${ }^{\star a}$ L. Malfatti, $^{b}$ D. Carboni, ${ }^{b}$ V. Alzari, ${ }^{c}$ A. Mariani ${ }^{c}$ and M. F. Casula*a
}

Graphene/silica nanocomposites in the form of highly porous aerogels are obtained for the first time by integrating a novel approach for the production of low defectivity graphene with a two-step route for the synthesis of a silica-based monolith. Different from the other synthetic methods, the use of cogelation of a dispersed phase and matrix followed by high temperature supercritical drying leads to well dispersed bilayered graphene inside a high surface area silica matrix with an open texture porosity. Physico-chemical characterization provides evidence that the developed graphene $/ \mathrm{SiO}_{2}$ bulk aerogel nanocomposites combine the distinct features of both the dispersed graphene sheets and the porous silica aerogel matrix. It was found that incorporation of graphene in the aerogel, even at low loading, increases significantly the hydrophobic behaviour of the materials. This, combined with the high surface/ volume ratio of the aerogel, makes the resulting nanocomposite a suitable candidate as a novel oil sorbent for water remediation. In particular, the developed graphene/silica aerogels selectively and quickly uptake oil, up to more than 7 times the aerogel sorbent mass, from oil-water mixtures, and keeps floating on water after absorbing the oil phase. The suitability of the developed composites as a class of novel sorbents for environmental remediation in the occurrence of flammable liquid spills, where burning represents a major threat, is supported by the specific features of silica aerogels such as a relative fire-resistance, in addition to the high porosity and hydrophobic nature.

Received 13th April 2016

Accepted 30th June 2016

DOI: $10.1039 / c 6 r a 09618 b$

www.rsc.org/advances reference to corals and fish eggs. ${ }^{2}$ As a consequence, intense research has recently focused on the use of sorbents. ${ }^{3}$ In particular, hydrophobic and oleophilic materials, due to their ability to repel water and absorb oils or other organic solvents with high volume and rate, have a major role as prospective tools for serious environmental issues. ${ }^{4}$ Sol-gel techniques have been found to be particularly suited for the design of hydrophobic and oleophilic sorbents, as the soft chemistry conditions enable the incorporation of non polar organic moieties either during or after inorganic network formation. ${ }^{5}$ In addition, sol-gel protocols which enable a fine control over the textural features responsible for sorption properties such as surface area, pore size, shape, symmetry, and interconnection have now been successfully established. ${ }^{6-8}$

Silica aerogels are to date the solids with the largest porosities and can be produced by sol-gel routes which include specific drying procedures that do not affect the high porosity associated to the open and branched solid network formed in the wet gel.9,10 The combination of large porosity and hydrophobicity has been shown to make aerogels technologically relevant materials in a broad range of applications from thermal insulation, ${ }^{\mathbf{1 1}, \mathbf{1 2}}$ to drug delivery, ${ }^{\mathbf{1 3}}$ and Cherenkov detector $^{\mathbf{1 4}}$ and their development still attracts a lively interest. ${ }^{\mathbf{1 5}}$ The sorption properties of silica aerogels, associated to their high surface area and porosity, are well documented. Since the pioneering work by Hrubesh et al., ${ }^{16}$ extensive use of 
hydrophobic silica aerogel in environmental clean-up, ${ }^{17,18}$ and particularly in oil spill-up, has been made. ${ }^{19-21}$

In this work, we address the design of a sorbent for prospective application in water clean-up treatment by introducing for the first time a composite aerogel with both hydrophobic and oleophilic features made out of exfoliated graphene sheets dispersed into highly porous amorphous silica. Graphene, featuring two-dimensional single atom carbon sheets, has recently inspired intense research because of its peculiar physicochemical features. ${ }^{22-24}$ For instance, electrical, physical, chemical, mechanical, and structural properties ${ }^{25-27}$ have been proposed for applications in fields such as nanoelectronics, ${ }^{28-31}$ sensing, ${ }^{32}$ clean energy devices, ${ }^{22-24,33}$ catalysis $^{34}$ and decontamination of wastewater. ${ }^{35,36}$ Within this framework, the hydrophobic properties of graphene have been recently exploited for oil absorption..$^{37-41}$ It has been recognized, however, that a key issue to fully exploit the application capabilities of graphene in environmental issues lies in either its assembly into 3D structures or incorporation into a matrix, ${ }^{42}$ and novel composites where the synergistic occurrence of graphene dispersed in a suitable matrix has lead to mixed functionalities have been proposed for use in solar energy conversion and removal of pollutants. ${ }^{43,44}$ Silica in particular is regarded as a suitable matrix due to intrinsic physico-chemical properties, including being relatively inert and non-toxic, which makes it suitable for environmental remediation. ${ }^{45,46}$

Here, the morpho-structural features of the novel graphene/ silica aerogels were investigated with particular emphasis onto the effect of graphene loading on the hydrophobic/oleophilic properties and ultimately on oil sorption efficiency of the nanocomposites.

\section{Experimental section}

\section{Preparation of graphene in DMF dispersion}

Graphene was obtained using a method recently optimized by Mariani et al., ${ }^{47-51}$ based on the exfoliation of graphite ${ }^{52}$ in a liquid medium (solvent or reactive medium) to form single or few-layer graphene. In this work, graphene was obtained in $\mathrm{N}, \mathrm{N}$ dimethylformamide (DMF, Sigma Aldrich, MW: 73.08, bp: 152$\left.154{ }^{\circ} \mathrm{C}\right)$. In detail, graphite flakes $(5.0 \mathrm{~g}$, particle size +100 mesh ( $\geq 75 \% \mathrm{~min}$ ), Sigma Aldrich) dispersed in $100 \mathrm{~g}$ of DMF were introduced in a $250 \mathrm{~mL}$ flask; the mixture was sonicated in an ultrasonic bath at room temperature for $24 \mathrm{~h}$. The dispersion was then centrifuged for $30 \mathrm{~min}$ at $4000 \mathrm{rpm}$, and the residual solid graphite was removed. The concentration, calculated by gravimetry after filtration through polyvinylidene fluoride filters (pore size $0.22 \mu \mathrm{m}$ ), was found to be equal to $0.35 \mathrm{mg} \mathrm{mL}^{-1}$.

\section{Synthesis of graphene/silica aerogel nanocomposites}

The nanocomposite sorbents were prepared using a modification of a synthetic approach developed in our laboratories for the preparation of silica-based aerogels. ${ }^{53-55}$ In a typical synthesis, $3.95 \mathrm{~mL}$ of tetraethoxysilane $\left(\mathrm{Si}\left(\mathrm{OC}_{2} \mathrm{H}_{5}\right)_{4}\right.$, Aldrich, $98 \%$, TEOS) which acts as silica precursor are added to absolute ethanol (EtOH, Fluka) and pre-hydrolyzed under acidic conditions using $1.965 \mathrm{~mL}$ of acidic stock hydrolyzing solution (prepared by mixing $2 \mathrm{~mL}$ of nitric acid $\left(\mathrm{HNO}_{3}\right.$, Carlo Erba, $65 \%), 80 \mathrm{~mL}$ of absolute ethanol and $130 \mathrm{~mL}$ of distilled water). To obtain a final nanocomposite with a graphene loading of 0.1 wt\% graphene/(graphene $+\mathrm{SiO}_{2}$ ), $2.985 \mathrm{~mL}$ of graphene dispersion in DMF were added into the pre-hydrolyzed TEOS under acidic catalysis. A hydro-alcoholic solution of urea $\left(\mathrm{NH}_{2} \mathrm{CONH}_{2}\right.$, Aldrich, $\left.>99.0 \%\right)$ was then added and kept under reflux at $85{ }^{\circ} \mathrm{C}$. Few drops of a solution of lithium borohydride $\left(\mathrm{LiBH}_{4}, 2.0 \mathrm{M}\right.$ solution in THF, Aldrich) were added after 40 min, giving rise to gelation of the sol after less than $1 \mathrm{~min}$. The gel was kept in oven at $40{ }^{\circ} \mathrm{C}$ for $3 \mathrm{~h}$ and then submitted to high temperature supercritical drying (up to $330{ }^{\circ} \mathrm{C}, 70 \mathrm{~atm}$ ) in an autoclave (Parr, $300 \mathrm{~cm}^{3}$ ) filled with $70 \mathrm{~mL}$ of absolute ethanol. The amount of graphene dispersion was varied in order to obtain different graphene loadings.

\section{Physical-chemical characterization}

Transmission electron microscopy (TEM) images were recorded on a Hitachi $\mathrm{H}-7000$ instrument running at $125 \mathrm{kV}$ and equipped with a AMT DVC $(2048 \times 2048$ pixel $)$ CCD camera. Prior to observation, the samples were finely ground and deposited on a carbon-coated copper grid.

Surface areas, pore sizes and pore volumes were obtained from $\mathrm{N}_{2}$ adsorption-desorption measurements at $77 \mathrm{~K}$ recorded on a Micromeritics ASAP2020. Surface area was estimated using the Brunauer-Emmett-Teller (BET) model, pore size and pore volumes were estimated using the Barrett-Joyner-Halenda (BJH) method. ${ }^{56,57}$

FTIR spectra were obtained through a Bruker Tensor 27 spectrometer (250 scans; resolution $2 \mathrm{~cm}^{-1}$ ) at $25{ }^{\circ} \mathrm{C}$. The collected spectra were manipulated using OPUS (version 6.5) software. In particular, a Platinum-ATR accessory and a DTGS (deuterated tri-glycine sulfate) detector were used.

Raman analyses were performed on powders of aerogel samples deposited onto silicon wafer by using a Bruker Senterra confocal Raman microscope with a laser excitation wavelength of $532 \mathrm{~nm}, 12.5 \mathrm{~mW}$ of nominal power and a $50 \times$ objective. The spectra were recorded in the $70-4500 \mathrm{~cm}^{-1}$ range, with a resolution of $9 \mathrm{~cm}^{-1}$, an integration time of $3 \mathrm{~s}$ and 6 co-additions. The number of graphene layers was estimated through a Laurentian fit of the Raman spectra in the $2820-2560 \mathrm{~cm}^{-1}$ range by using an analysis software. The fit provided a $R^{2}$ higher than 0.9995 .

The hydrophobicity of the samples was evaluated using a Dataphysics OCA 20 instrument by measuring the contact angle of $5 \mu \mathrm{L}$ water droplets deposited onto aerogels fragments. The reported contact angle was taken as a median of at least 5 measurements.

\section{Adsorption capacity measurements}

The adsorption tests were performed by placing a block of nanocomposite aerogel, with a given mass $m_{\mathrm{a}}$, in a mineral oil (Aldrich, viscosity $>34.7 \mathrm{cSt}$, density $0.862 \mathrm{~g} \mathrm{~mL}^{-1}$ ) or crude oil (Sartec, viscosity $<20.5 \mathrm{cSt}$, density $<1.010 \mathrm{~g} \mathrm{~mL}^{-1}$ ) or in 
a mixture of distilled water and mineral oil colored by Oil Blue $\mathrm{N}$ (Aldrich).

\section{Results and discussion}

\section{Design and characterization of the aerogel nanocomposites}

Graphene/silica nanocomposites were obtained for the first time in the form of aerogels. The sol-gel procedure was designed to incorporate a graphene suspension at the early stages of gel formation, ensuring homogeneous dispersion of graphene within the silica network, followed by supercritical drying of the wet gel. The issue related to the incorporation of graphene nanosheets into a silica matrix through sol-gel chemistry is mainly due to the low dispersibility of graphene in most common solvents. ${ }^{58}$ Several kinds of common graphene dispersants have been explored, such as poly(ethyleneglycol) diacrylate and 1-methyl-2-pyrrolidone, ${ }^{59}$ as well as dispersants successfully employed in the production of graphene nanocomposite by sol-gel such as 1-vinyl-2-pyrrolidone. ${ }^{60-62}$ These dispersants, however, turned out to produce a final aerogel nanocomposite with highly inhomogeneous morphology, probably due to polymerization of the dispersant at the relatively high temperatures and pressures employed during supercritical drying. Finally, the combined choice of DMF, as dispersant, and $\mathrm{LiBH}_{4}$, as reducing agent, enabled us to obtain a well-dispersed nanocomposite with hydrophobic properties. It should be pointed out that, without using $\mathrm{LiBH}_{4}$, the resulting nanocomposite keeps indeed its high porosity and homogeneity, but shows hydrophilic features (see ESI, Fig. S1-S3†). On the other hand, through the optimized synthetic approach, aerogels with increased hydrophobicity provided by the graphene addition were obtained for the first time. In particular, Fig. 1a shows a water droplet deposited on the surface of the 0.1 wt\% graphene/aerogel nanocomposite where the quasispherical shape of the droplet evidences the extreme water repellency.

The strong hydrophobic character is confirmed by contact angle measurements (Fig. 1b), which provide an average contact angle value of $144^{\circ}$, higher than those of undoped silica aerogel $\left(138^{\circ}\right)$, suggesting a valuable change of the aerogel functional properties even at very low graphene loadings. We ascribe the high contact angle to the combined hydrophobic features of the nanocomposite and to the textural features of aerogels and resulting surface roughness. In particular, when the contact angle is measured on the graphene/silica $0.01 \mathrm{wt} \%$ nanocomposite aerogel pressed into a dense pellet, the value for the same sample decreases from $144^{\circ}$ in the form of aerogel to $75^{\circ}$ in the form of dense pellet, indicating that the hydrophobic properties of our graphene/silica aerogel arise from the combined contribution of graphene and of the texture of aerogels.

TEM investigation of the graphene/silica nanocomposites (Fig. 1c-e) shows a necklace silica network with the characteristic porous texture of aerogels, which encloses well dispersed graphene sheets. SEM images support the above findings, indicating the occurrence of layered structures due to graphene sheets dispersed within the mesoporous matrix (Fig. S5†).
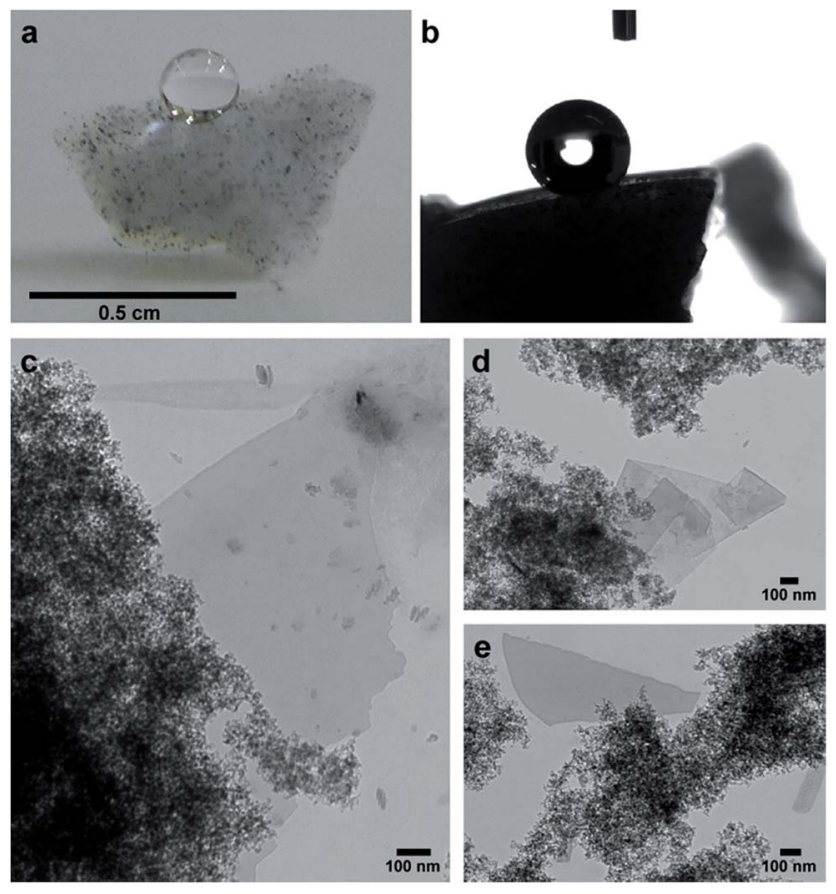

Fig. 1 Digital image of a water droplet on the surface of a block of hydrophobic graphene/silica aerogel nanocomposite (a) and representative image used for water contact angle measurements (b). TEM images of the graphene/silica aerogel nanocomposite $(c-e)$.

These observations suggest that the proposed procedure is effective in introducing graphene sheets within the silica aerogel and that the chemicals adopted for the sol-gel synthesis of the graphene/silica nanocomposite do not affect the textural features typical of silica aerogels.

The porous structure of the aerogel nanocomposites has been analyzed through $\mathrm{N}_{2}$ physisorption. Fig. S6 $\dagger$ shows a type IV isotherm with a hysteresis lying at high relative pressures for the graphene/silica nanocomposites as well as for the pure silica aerogel. This isotherm is typical of aerogels with interconnected and open large pores (with average size $30 \mathrm{~nm}$ ) suitable for adsorption. The addition of graphene reduces the available surface area of the silica matrix from $530 \mathrm{~m}^{2} \mathrm{~g}^{-1}$ (undoped aerogel) down to $473 \mathrm{~m}^{2} \mathrm{~g}^{-1}$ (graphene/silica materials $0.1 \mathrm{wt} \%)$.

As Raman spectroscopy was demonstrated to be a valuable tool to gain insights on the quality and aggregation state of graphene, the Raman spectra of the nanocomposite aerogels were collected, as shown in Fig. 2 for the nanocomposite aerogel with a loading of $0.1 \mathrm{wt} \% .^{60,63,64}$

The spectrum is dominated by a narrow and intense band peaked at $1568 \mathrm{~cm}^{-1}$ (G band) associated to the first-order scattering of the $\mathrm{E}_{2 \mathrm{~g}}$ mode of $\mathrm{C} \mathrm{sp}^{2}$ atoms. Additional features include a band peaking at $1340 \mathrm{~cm}^{-1}$ (D band) related to the breathing mode of $k$-point phonons of $\mathrm{A}_{1 \mathrm{~g}}$ symmetry and a band centered at $2685 \mathrm{~cm}^{-1}$ (2D) being the first overtone of the D band, ascribed to the sum of two phonons with opposite momenta. The $\mathrm{D}^{\prime}$ band at $1608 \mathrm{~cm}^{-1}$, attributed to Stokes scattering by a longitudinal optical phonon, is overwhelmed by the $\mathrm{G}$ band almost looking as a shoulder. All the above features, 


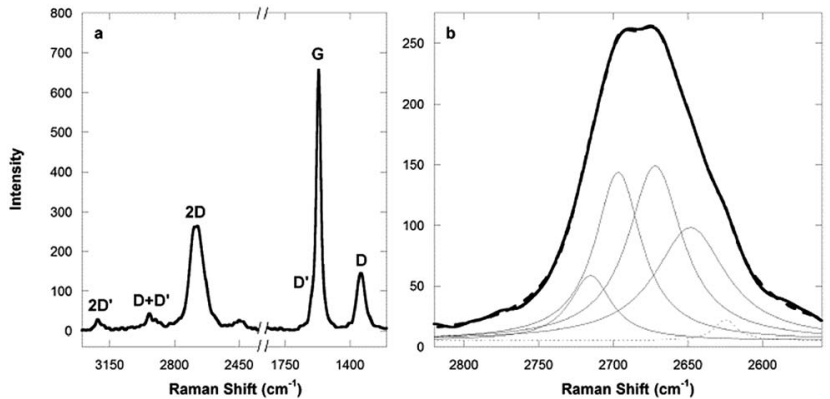

Fig. 2 Raman spectrum of the hydrophobic graphene/ $/ \mathrm{SiO}_{2}$ aerogel nanocomposite (a) and detail of the $2820-2560 \mathrm{~cm}^{-1}$ range (b): bold curve is the acquired Raman spectrum, bold dotted line describes the fitting obtained by combining the Lorentzian curves (thin line), and thin dotted lines are the fitted band of the silicon signal.

together with the first overtone of the $\mathrm{D}^{\prime}$ band peaking at 3213 $\mathrm{cm}^{-1}\left(2 \mathrm{D}^{\prime}\right)$ and the $\mathrm{D}+\mathrm{D}^{\prime}$ band centered at $2935 \mathrm{~cm}^{-1}$, are indicative of the occurrence of graphene. ${ }^{60}$

Peak profile analysis of the 2D Raman band centered at about $2685 \mathrm{~cm}^{-1}$ was performed to assess the aggregation state of the exfoliated graphene flakes within the aerogel nanocomposite. The number of Lorentzian curves capable of fitting the $2 \mathrm{D}$ band can, in fact, clarify the nature of the layered structure (mono- or $n$-layers) of the embedded graphene. ${ }^{63,64} \mathrm{In}$ the case of our graphene/silica aerogels it was found that the 2D graphene could be effectively deconvoluted by 4 Lorentzian curves, as shown in Fig. 2b, suggesting the occurrence of the graphene flakes embedded into the silica aerogel matrix as bilayers and ruling out significant aggregation.

\section{Adsorption capacity measurements}

The potential of the developed graphene/ $\mathrm{SiO}_{2}$ aerogels as sorbents for oils was first tested on a mixture of distilled water and a commercial blue-coloured mineral oil, as shown in Fig. 3. Mineral oil is not miscible with water (Fig. 3a) and when a small fragment of graphene/ $/ \mathrm{SiO}_{2}$ aerogel is added, it floats on top of the water surface and goes into contact with the oil phase (Fig. 3b). The aerogel readily adsorbs the oil phase (Fig. 3c) and leaves the water surface clean once the oil-loaded aerogel is removed (Fig. 3d). The process is so quick that, under our standard experimental conditions, the adsorption goes to completion in a time scale of tens of seconds. During this process, no water absorption is observed suggesting that graphene induces an oil-selective adsorption property in these nanocomposites.

In order to investigate the sorption rate of the mineral oil into the aerogel, a standardized clean-up process of an oilcontaminated water was monitored as a function of time. In detail, a fragment of graphene-silica aerogel was placed on the surface of a water/oil mixture and the adsorption capacity $(k)$ defined as mass of oil-loaded aerogel to initial mass of the aerogel was measured at increasing times. The mass of mineral oil has been optimized and kept constant so that the mineral oil was never completely adsorbed from water. This ensures that all the samples were fully loaded at the end of the process.

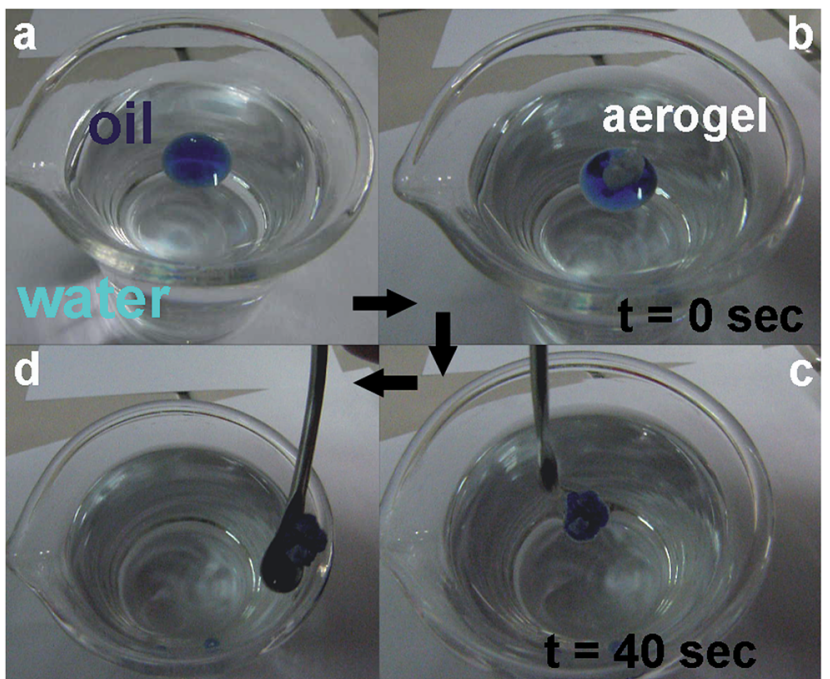

Fig. 3 Photographic sequence of the removal process of mineral oil, detectable as a blue liquid (a), from a water-oil mixture by $0.1 \mathrm{wt} \%$ graphene $/ \mathrm{SiO}_{2}$ aerogel nanocomposite (b). The aerogel completely adsorbs the oil phase (c) leaving only clean water (d).

Moreover, using this amount of oil we have not observed appreciable variations in the absorption rate of samples with similar composition (i.e. same graphene content) but different weight. Fig. 4 shows a plot of the adsorption capacity at different times; each point is the average value of the adsorption capacity collected in three different test using different blocks of the same sample. The adsorption capacity values increase rapidly over the first 30 seconds and the maximum of adsorption is

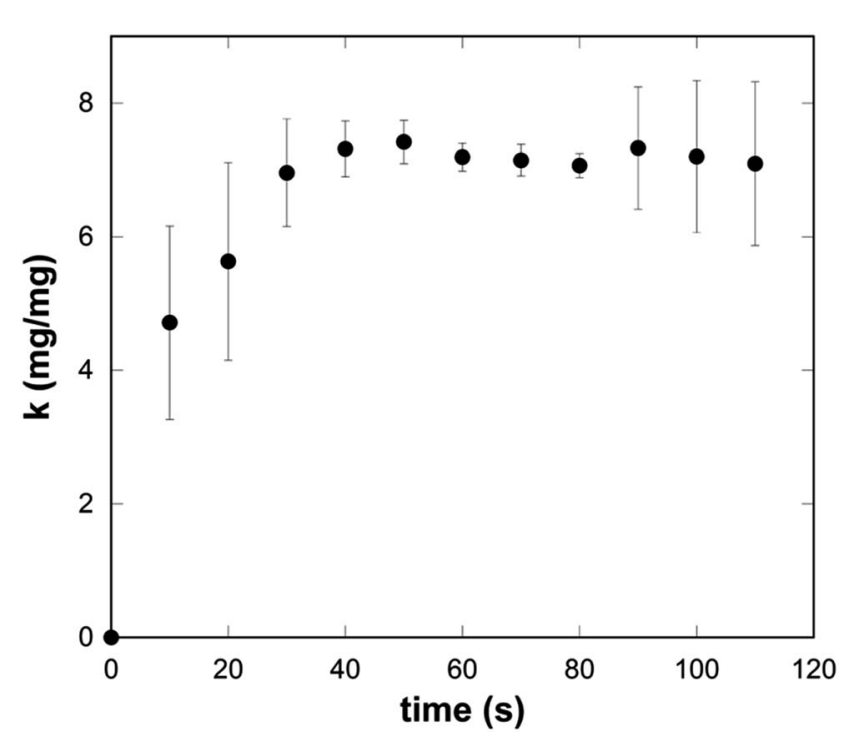

Fig. 4 Adsorption capacity of mineral oil from water by 0.1 wt\% graphene $/ \mathrm{SiO}_{2}$ aerogel nanocomposite. Each point is the average value of three determinations of the adsorption capacity. The relatively large error bar at the early steps of adsorption is due to the fast kinetic for oil uptake; the error in the plateau-regime zone is ascribed to the loss of very small aerogel fragments due to repeated mechanical dipping and withdrawal of the aerogel from the oil/water mixture. 
achieved after 40-50 seconds, after which the curve exhibits a plateau up to 120 seconds.

A quantitative assessment of the absorption capacity as a function of the graphene content $(0,0.003$ and $0.1 \mathrm{wt} \%)$ was performed by placing a fragment of nanocomposite (5-15 $\mathrm{mg}$ ) on the surface of the mineral oil. The aerogel was kept on the oil surface for 3 minutes assuming that, after this time, the maximum of adsorption was reached as resulted from the adsorption capacity experiments. All the three samples are hydrophobic and show very similar pore textures although different surface area (see Fig. S6†), which decreases when the graphene content increases (Table 1).

The adsorption capacities, $k$, of the nanocomposites with different graphene loading (0, 0.003 and $0.1 \mathrm{wt} \%)$ are reported in Fig. 5a, where every point is the average value of the adsorption capacity collected in five independent tests using different blocks of the same sample. Despite the very low quantity, graphene improves the adsorption properties of the aerogel, with an increase of the adsorption capacity of more than $20 \%$ for the $0.1 \mathrm{wt} \%$ nanocomposite as compared to the undoped aerogel.

This effect is even more remarkable if we take into account the reduced porosity (Fig. 5b); in fact by taking into account the different surface areas of the aerogels, the improvement of the performances provided by the graphene addition increases up to $35 \%$ for the $0.1 \mathrm{wt} \%$ nanocomposite. Preliminary tests on the adsorption capacity of crude oil from the graphene/silica aerogel with $0.01 \mathrm{wt} \%$ content show that values around 7 are obtained, supporting the potential of the materials for water remediation from oil spills.

The adsorption capacities observed for our aerogels are close to those found for silica aerogels ${ }^{16,19-21}$ and polyurea aerogel; ${ }^{65}$ however, it should be pointed out that comparison of the performance of different sorbents through their adsorption capacity values is not straightforward as the estimated values depend on several experimental conditions (determination on either powdered or monolithic sorbent; kind of the oil used; procedure used for the removal of the oil on the external surface prior to weighing, etc.).

On the other hand, it is noteworthy to remind that pure carbon-based materials have recently emerged as sorbents with extremely high $k$ values, such as graphene sponges ( $k$ values up to 100$),{ }^{38,39}$ foams (200), ${ }^{37}$ aerogels (250), ${ }^{40,41,66}$ and carbon aerogels (200). ${ }^{67}$ However, while pure carbon-based materials exhibit unprecedented oleophilic properties, it is expected that application of carbon/silica nanocomposite aerogels to environmental remediation may benefit from the use of

Table $1 N_{2}$ physisorption results for the aerogel samples: surface area $\left(S_{\mathrm{BET}}\right)$, pore volume $\left(V_{p}\right)$, and pore size $(d)$. Note that differences in values obtained from repeated runs were found to be less than $3 \%$

\begin{tabular}{llll}
\hline Graphene loading $(\mathrm{wt} \%)$ & $S_{\mathrm{BET}}\left(\mathrm{m}^{2} \mathrm{~g}^{-1}\right)$ & $V_{\mathrm{p}}\left(\mathrm{cm}^{3} \mathrm{~g}^{-1}\right)$ & $d(\mathrm{~nm})$ \\
\hline 0 & $530 \pm 2$ & 5.16 & 30 \\
0.003 & $493 \pm 2$ & 4.46 & 30 \\
0.1 & $473 \pm 3$ & 4.23 & 30
\end{tabular}

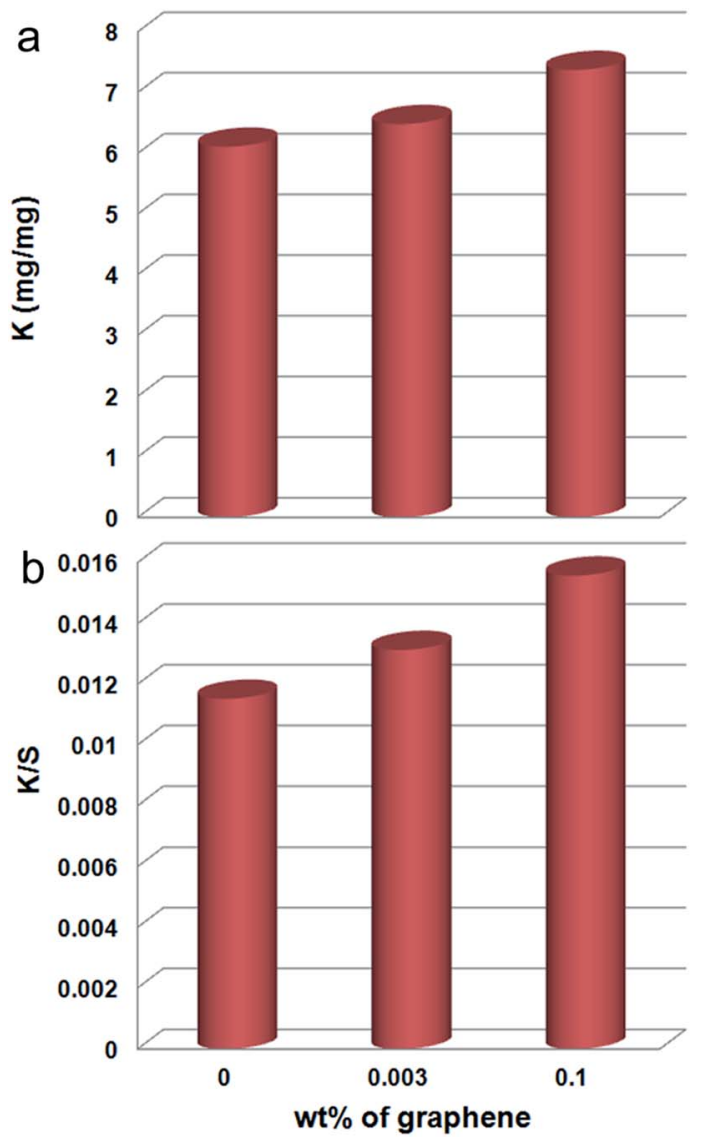

Fig. 5 Adsorption capacities of mineral oil by undoped $\mathrm{SiO}_{2}$ aerogel and by graphene $/ \mathrm{SiO}_{2}$ aerogel nanocomposites with different graphene loading after a contact time of 3 minutes ( $k$ error \pm 0.6$)(a)$ and $k$ values normalized for the surface area (b).

a lightweight though solid sorbent based on an inert and nontoxic material, such as silica aerogel.

A specific advantage associated to the use of silica-based carbon-doped sorbents can be found in the achievement of fire-resistance, as burning represents a major threat connected to oil spills. As shown in Fig. 6, the developed graphene/silica nanocomposite aerogels exhibit a relatively good heat resistance to fire which can be ascribed to the outstanding insulating properties of silica aerogels, and are therefore suited for use in the occurrence of flammable liquid spills. After oil uptake and burning, the graphene/silica nanocomposite aerogel still retains its hydrophobic features and keeps floating on water, although its oil adsorption capacity drops down to $50 \%$ of its original value (see ESI, par.S4 and Fig. $\mathrm{S} 7 \dagger$ for more details).

We prospect that the developed method for the synthesis of graphene/silica nanocomposite aerogels will open up the possibility to develop novel silica-based aerogel sorbents with improved performance for oil removal. It should be pointed out that successful preparation of composite aerogels by co-gelation of the dispersed and matrix phases, which ensures compositional homogeneity of the resulting material, requires the use of high temperature supercritical drying techniques such as the one adopted here. In fact, although less expensive drying 

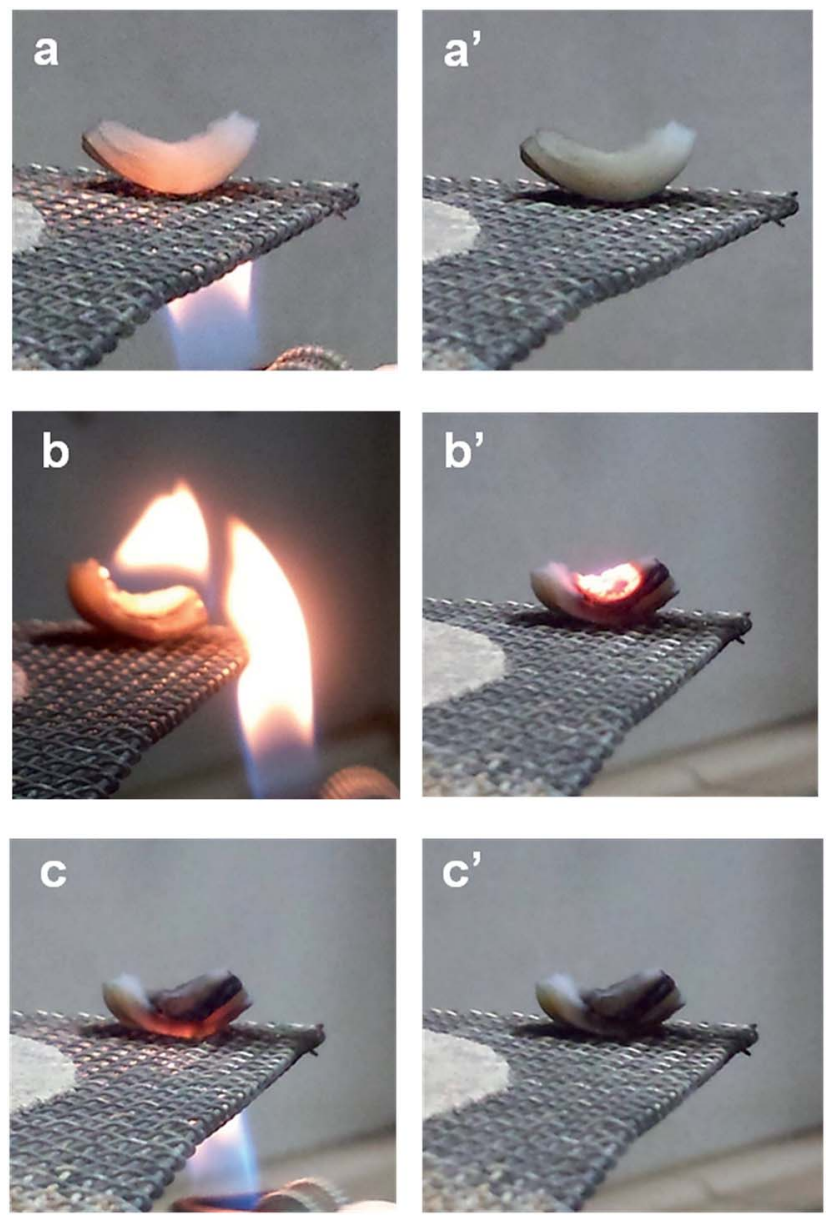

Fig. 6 Digital images showing the effect of direct flame on a graphene/silica aerogel: the graphene/silica aerogel exposed to a flame (a) remains unaltered $\left(a^{\prime}\right)$. On the other hand, after the aerogel has been soaked in oil and is exposed to fire (b) it does burn, although burning is relatively controlled $\left(b^{\prime}\right)$ and when the aerogel is exposed again to a flame no variation is observed (c and $c^{\prime}$ ).

procedures would be also available to produce aerogel and aerogel-like materials, they all rely on solvent-exchange procedures which result in leakage of the dispersed phase from the wet gel. ${ }^{68}$ It is expected therefore that higher graphene loadings could be obtained without damaging the silica aerogel texture, as opposed to what observed in composites where graphene is added to the porous sorbent by repetitive post functionalization, leading to pore blocking at relatively high carbon loading. ${ }^{39}$

\section{Conclusions}

A nanocomposite aerogel made out of graphene sheets dispersed into amorphous silica has been obtained and tested as a novel sorbent for prospective application in water clean-up treatments. The use of suitable dispersants for the graphene phase in the sol, enables obtaining a composite gel, which was converted into a highly porous aerogel by supercritical drying, through a one-pot approach. The nanocomposite aerogels retain both the high quality of the initial bilayered graphene, dispersed in DMF, as well as the highly porous texture of typical silica aerogel matrix. Graphene loading as low as $0.003 \mathrm{wt} \%$ leads to graphene-silica aerogels with enhanced hydrophobic and oleophilic features, as demonstrated by water contact angle measurements and commercial mineral oil uptake. The efficiency of prepared graphene/silica nanocomposite aerogels for oil spill clean-up were assessed through water-oil separation experiments. Small amount of graphene $(0.1 \mathrm{wt} \%)$ in the nanocomposite induces a relative increase of the absorption property by $20 \%$ compared to undoped silica aerogel; however, if the absorption property is rescaled by the surface area, the relative increase is enhanced up to $35 \%$. Thanks to the versatility of the sol-gel chemistry, we expect to extend our innovative approach to a broader range of compositions, in order to produce optimized sorbents for environmental remediation.

We anticipate that the route developed here for graphene/ silica aerogels will inspire novel protocols for the integration of graphene into nanocomposites, expanding the applicability of graphene properties and opening up the way to the design of novel multifunctional materials.

\section{Acknowledgements}

Drs S. Puligheddu and M. Meloni from Sartec SpA are gratefully acknowledged for kindly providing crude oil samples. Regione Autonoma della Sardegna under LR7/2007 and Fondazione Banco di Sardegna are acknowledged for financial support. Dr F. Cugia and Dr S. Podda are acknowledged for access and support in FTIR and SEM measurements at the Scientific Park "Sardegna Ricerche". L.M. and D.C. acknowledge the Italian Ministry of Foreign Affairs and International Cooperation (MAECI) for funding through the cooperation project "GRAPEMAT” (PGR04266).

\section{References}

1 ExxonMobil, Oil spill response field manual, http:// cdn.exxonmobil.com/ /media/global/files/other/2015/oilspill-response-field-manual_2014_e.pdf.

2 J. Q. Word, J. R. Clark and L. S. Word, Hum. Ecol. Risk Assess., 2015, 21, 707-725.

3 M. N. Rashed, Adsorption Technique for the Removal of Organic Pollutants from Water and Wastewater, in Organic Pollutants - Monitoring, Risk and Treatment, ed. M. N. Rashed, InTech, 2013, pp. 167-194.

4 M. O. Adebajo, R. L. Frost, J. T. Kloprogge, O. Carmody and S. Kokot, J. Porous Mater., 2003, 10, 159-170.

5 P. Falcaro, S. Costacurta, L. Malfatti, D. Buso, A. Patelli, P. Schiavuta, M. Piccinini, G. Grenci, B. Marmiroli, H. Amenitsch and P. Innocenzi, ACS Appl. Mater. Interfaces, 2011, 3, 245-251.

6 K. Nakanishi and N. Tanaka, Acc. Chem. Res., 2007, 40, 863873.

7 C. Sanchez, K. J. Shea and S. Kitagawa, Chem. Soc. Rev., 2011, 40, 471-472.

8 C. Triantafillidis, M. S. Elsaesser and N. Hüsing, Chem. Soc. Rev., 2013, 42, 3833-3846. 
9 A. C. Pierre and G. M. Pajonk, Chem. Rev., 2002, 102, 42434265.

10 T. Tillotson and L. W. Hrubesh, J. Non-Cryst. Solids, 1992, 145, 44-50.

11 J. Fesmire, Cryogenics, 2006, 46, 111-117.

12 G. Hayase, K. Kanamori, K. Abe, H. Yano, A. Maeno, H. Kaji and K. Nakanishi, ACS Appl. Mater. Interfaces, 2014, 6, 94669471.

13 I. Smirnova, J. Mamic and W. Arlt, Langmuir, 2003, 19, 85218525.

14 A. Gougas, D. Ilie, S. Ilie and V. Pojidaev, Nucl. Instrum. Methods Phys. Res., 1999, 421, 249-255.

15 W. J. Malfait, S. Zhao, R. Verel, S. Iswar, D. Rentsch, R. Fener, Y. Zhang, B. Milow and M. M. Koebel, Chem. Mater., 2015, 27, 6737-6745.

16 L. W. Hrubesh, P. R. Coronado and J. H. Satcher Jr, J. NonCryst. Solids, 2001, 285, 328-332.

17 M. L. N. Perdigoto, R. C. Martins, N. Rocha, M. J. Quina, L. Gando-Ferreira, R. Patricio and L. Duraes, J. Colloid Interface Sci., 2012, 380, 134-140.

18 S. Štandeker, Z. Novak and Ž. Knez, J. Colloid Interface Sci., 2007, 310, 362-368.

19 J. G. Reynolds, P. R. Coronado and L. W. Hrubesh, Energy Sources, 2001, 23, 831-843.

20 J. G. Reynolds, P. R. Coronado and L. W. Hrubesh, J. NonCryst. Solids, 2001, 292, 127-137.

21 A. Venkateswara Rao, N. D. Hegde and H. Hirashima, J. Colloid Interface Sci., 2007, 305, 124-132.

22 W. Cai, Y. Zhu, X. Li, R. D. Piner and R. S. Ruoff, Appl. Phys. Lett., 2009, 95, 123115.

23 A. Reina, X. Jia, J. Ho, D. Nezich, H. Son, V. Bulovic, M. S. Dresselhaus and J. Kong, Nano Lett., 2009, 9, 30-35.

24 Y. Zhu, S. Murali, W. Cai, X. Li, J. W. Suk, J. R. Potts and R. S. Ruoff, Adv. Mater., 2010, 22, 3906-3924.

25 D. C. Wei and Y. Q. Liu, Adv. Mater., 2010, 22, 3225-3241.

26 C. Lee, X. Wei, J. W. Kysar and J. Hone, Science, 2008, 321, 385-388.

27 A. A. Balandin, S. Ghosh, W. Bao, I. Calizo, D. Teweldebrhan, F. Miao and C. N. Lau, Nano Lett., 2008, 8, 902-907.

28 X. Zhang, Y. Lai, M. Ge, Y. Zheng, K.-Q. Zhang and Z. Lin, J. Mater. Chem. A, 2015, 3, 12761-12768.

29 X. Wu, J. Zhou, W. Xing, G. Wang, H. Cui, S. Zhuo, Q. Xue, Z. Yana and S. Z. Qiao, J. Mater. Chem., 2012, 22, 2318623193.

30 G. D. Moon, J. B. Joo and Y. Yin, Nanoscale, 2013, 5, 1157711581.

31 D. Wang, X. Liu, L. He, Y. Yin, D. Wu and J. Shi, Nano Lett., 2010, 10, 4989-4993.

32 Y. X. Liu, X. C. Dong and P. Chen, Chem. Soc. Rev., 2012, 41, 2283-2307.

33 T. Umeyama and H. Imahori, J. Phys. Chem. C, 2013, 117, 3195-3209.

34 L. Shang, T. Bian, B. Zhang, D. Zhang, L.-Z. Wu, C.-H. Tung, Y. Yin and T. Zhang, Angew. Chem., Int. Ed., 2014, 53, 250254.
35 H. Wang, X. Yuan, Y. Wu, H. Huang, X. Peng, G. Zeng, H. Zhong, J. Liang and M. Ren, Adv. Colloid Interface Sci., 2013, 195, 19-40.

36 S. Wang, H. Sun, H. M. Ang and M. O. Tadé, Chem. Eng. J., 2013, 226, 336-347.

37 X. Dong, J. Chen, Y. Ma, J. Wang, M. B. Chan-Park, X. Liu, L. Wang, W. Huang and P. Chen, Chem. Commun., 2012, 48, 10660-10662.

38 H. Bi, X. Xie, K. Yin, Y. Zhou, S. Wan, L. He, F. Xu, F. Banhart, L. Sunand and R. S. Ruoff, Adv. Funct. Mater., 2012, 22, 44214425.

39 D. D. Nguyen, N.-H. Tai, S.-B. Lee and W.-S. Kuo, Energy Environ. Sci., 2012, 5, 7908-7912.

40 L. Xu, G. Xiao, C. Chen, R. Li, Y. Mai, G. Sun and D. Yan, J. Mater. Chem. A, 2015, 3, 7498-7504.

41 S. Liao, T. Zhai and H. Xia, J. Mater. Chem. A, 2016, 4, 10681077.

42 X. Chen and B. Chen, Environ. Sci. Technol., 2015, 49, 61816189.

43 H. Chang and H. Wu, Energy Environ. Sci., 2013, 6, 34833507.

44 P. Innocenzi, L. Malfatti and D. Carboni, Nanoscale, 2015, 7, 12759-12772.

45 T. Zheng, J. Zhan, J. He, C. Day, Y. Lu, G. L. McPherson, G. Piringer and V. T. John, Environ. Sci. Technol., 2008, 42, 4494-4499.

46 S. Kabiri, D. N. H. Tran, S. Azari and D. Losic, ACS Appl. Mater. Interfaces, 2015, 7, 11815-11823.

47 V. Alzari, D. Nuvoli, R. Sanna, L. Peponi, M. Piccinini, S. Bittolo Bon, S. Marceddu, L. Valentini, J. M. Kenny and A. Mariani, Colloid Polym. Sci., 2013, 291, 2681-2687.

48 S. Scognamillo, E. Gioffredi, M. Piccinini, M. Lazzari, V. Alzari, D. Nuvoli, R. Sanna, D. Piga, G. Malucelli and A. Mariani, Polymer, 2012, 53, 4019-4024.

49 V. Alzari, D. Nuvoli, R. Sanna, S. Scognamillo, M. Piccinini, J. M. Kenny, G. Malucelli and A. Mariani, J. Mater. Chem., 2011, 21, 16544-16549.

50 V. Alzari, D. Nuvoli, S. Scognamillo, M. Piccinini, E. Gioffredi, G. Malucelli, S. Marceddu, M. Sechi, V. Sanna and A. Mariani, J. Mater. Chem., 2011, 21, 8727-8733.

51 V. Alzari, V. Sanna, S. Biccai, T. Caruso, A. Politano, N. Scaramuzza, M. Sechi, M. Piccinini, D. Nuvoli, R. Sanna, S. Marceddu and A. Mariani, Composites, Part B, 2014, 60, 29-35.

52 M. Cai, D. Thorpe, D. H. Adamson and H. C. Schniepp, J. Mater. Chem., 2012, 22, 24992-25002.

53 M. F. Casula, D. Loche, S. Marras, G. Paschina and A. Corrias, Langmuir, 2007, 23, 3509-3512.

54 D. Loche, M. F. Casula, A. Falqui, S. Marras and A. Corrias, J. Nanosci. Nanotechnol., 2010, 10, 1008-1016.

55 A. Casu, M. F. Casula, A. Corrias, A. Falqui, D. Loche, S. Marras and C. Sangregorio, Phys. Chem. Chem. Phys, 2008, 10, 1043-1052.

56 S. Brunauer, P. H. Emmet and E. Teller, J. Am. Chem. Soc., 1938, 60, 309-319.

57 E. P. Barret, L. G. Joyner and P. P. Halenda, J. Am. Chem. Soc., 1951, 73, 373-380. 
58 P. Innocenzi, L. Malfatti, B. Lasio, A. Pinna, D. Loche, M. F. Casula, V. Alzari and A. Mariani, New J. Chem., 2014, 38, 3777-3782.

59 Y. Hernandez, V. Nicolosi, M. Lotya, F. M. Blighe, Z. Y. Sun, S. De, I. T. McGovern, B. Holland, M. Byrne, Y. K. Gun'Ko, J. J. Boland, P. Niraj, G. Duesberg, S. Krishnamurthy, R. Goodhue, J. Hutchison, V. Scardaci, A. C. Ferrari and J. N. Coleman, Nat. Nanotechnol., 2008, 3, 563-568.

60 L. Malfatti, P. Falcaro, A. Pinna, B. Lasio, M. F. Casula, D. Loche, A. Falqui, B. Marmiroli, H. Amenitsch, R. Sanna, A. Mariani and P. Innocenzi, ACS Appl. Mater. Interfaces, 2014, 6, 795-802.

61 D. Carboni, B. Lasio, V. Alzari, A. Mariani, D. Loche, M. F. Casula, L. Malfatti and P. Innocenzi, Phys. Chem. Chem. Phys., 2014, 16, 25809-25818.
62 D. Carboni, B. Lasio, D. Loche, M. F. Casula, A. Mariani, L. Malfatti and P. Innocenzi, J. Phys. Chem. Lett., 2015, 6, 3149-3154.

63 A. C. Ferrari, J. C. Meyer, V. Scardaci, C. Casiraghi, M. Lazzeri, F. Mauri, S. Piscanec, D. Jiang, K. S. Novoselov, S. Roth and A. K. Geim, Phys. Rev. Lett., 2006, 97, 187401.

64 L. M. Malard, M. A. Pimenta, G. Dresselhaus and M. S. Dresselhaus, Phys. Rep., 2009, 473, 51-87.

65 N. Leventis, C. Chidambareswarapattar, A. Bang and C. Sotiriou-Leventis, ACS Appl. Mater. Interfaces, 2014, 6, 6872-6882.

66 J. Li, J. Li, H. Meng, S. Xie, B. Zhang, L. Li, H. Ma, J. Zhanga and M. Yu, J. Mater. Chem. A, 2014, 2, 2934-2941.

67 R. J. White, N. Brun, V. L. Budarin, J. H. Clark and M.-M. Titirici, ChemSusChem, 2014, 7, 670-689.

68 A. Soleimani Dorcheh and M. H. Abbasi, J. Mater. Process. Technol., 2008, 199, 10-26. 